\title{
Real-Time Measurement and Monitoring of Industrial Pharmaceutical Chemical Reactor Process Parameters (Temperature, Level and Pressure) using PLC \& SCADA System
}

\author{
Mr. Lavanuru Ashok ${ }^{1}$ \\ Research Scholar, \\ Department of Instrumentation, \\ Sri Krishnadevaraya University, \\ Ananthapuramu, A.P., India-515003.
}

\author{
Prof. B. Rama Murthy 1 \\ Research Supervisor (Retd.), \\ Department of Instrumentation, \\ Sri Krishnadevaraya University, \\ Ananthapuramu, A.P., India-515003.
}

\begin{abstract}
Reactor is one of the most important equipment in pharmaceutical industry which requires continuous measurement and monitoring of process parameters like reactor mass temperature, reactor inner volume level and internal mass pressure of reactor. There are possibilities of errors in the measurements various stages involved with human workers. So advanced measurement and monitoring system is required to avoid failures, and are achieved by Programmable Logic Controller (PLC) and Supervisory Control And Data Acquisition system (SCADA). The present work chemical reactor consist of three different types of sensors, one is Resistive Temperature Detector (RTD) for measuring the reactor mass temperature, capacitive type level sensor for measuring the inner substances level and third one is diaphragm type pressure sensor used for measurement of internal pressure of reactor. SCADA is used for continuous visual monitoring of reactor temperature, pressure and Level sensors outputs given to a PLC. Which process all the process parameters of reactor. The SCADA system is conceptualized and developed to generate reports and trends of the data recorders in the SCADA system. The proposed system is capable of performing real-time measurement of industrial physical parameters data that can be efficiently transferred from PLC to a SCADA system which is stored and monitored through MODBUS communication protocol. It can be conclude from the theoretical data and experimental results data that proposed real-time measurement and monitoring achieved for Industrial pharmaceutical chemical reactor system.
\end{abstract}

Keywords: Industrial Pharmaceutical Chemical reactor, PLC, SCADA, Level sensor, Pressure sensor, Temperature sensor, MODBUS Comm. Protocol.

\section{INTRODUCTION}

The industrial pharmaceutical chemical reactors are extremely important because the output products are different types of drugs, chemicals, tablets, and pesticides have a great usage in our day to day life. Many researchers have studied that the usage of SCADA/PLC system are used in the boiler operation, distillation plants and water distribution plants. Now a day's industrial automation technology is well established in infrastructure systems for chemical processing reaction experiment, leads to heavy use of Programmable
Logic Controllers (PLC) which typically are intelligent automation stations forming the core of industrial systems [1] Hybrid wind-PV-battery renewable energy system is connected to PLC and the system is Real-time monitoring and control by SCADA and PLC. The total system consist of two induction generators (IG1\&IG2), solar system (PV), a AC-to-DC convertor, a battery unit, and DC-to-AC inverter with large number of inputs and outputs signals connected to the CSI series of OMRAN PLC connected with the SCADA system for monitoring system [2] water storage and distribution process are in pharmaceutical industries facing crucial to sustain community health, clean and safe environment purpose applying the SCADA solutions has a positive impact on the operations, maintains, process development and saving from water storage and distribution plants. A SCADA software application is implemented on the plants with interface to the hardware to connect a comprehensive real-time application environment for the modern water storage and distribution plants [3] industrial power plants must have steam boilers, so the boilers require continuous measuring, monitoring and inspections at frequent intervals there it is number of physical parameters like steam level temperature, boiler drum level and pressure of the steam line. The steam level temperature produces electrical power generation and steam water reuse different section in relevant temperatures. In order to automate a boiler plant and minimize the human intervals, there is need to develop PLC/SCADA system, SCADA is a centralized system used to supervise a complete plant. In boiler automation system which consists of PLC/SCADA, resistive temperature detector PT 100 (RTD PT 100) is used to measure the temperature, RT pressure switch is used to measure the pressure inside the boiler and float switch are used to detect the feed water level inside the boiler [4]. The focus of this paper is to proposed a real-time measurement, monitoring and reporting alarm system for measurement Temperature, Level and Pressure values of industrial pharmaceutical chemical reactor system 


\section{II.HARDWARE OF THE PRESENTED SYSTEM}

Fig.1 shows the Block diagram of L.T and TT of the process.

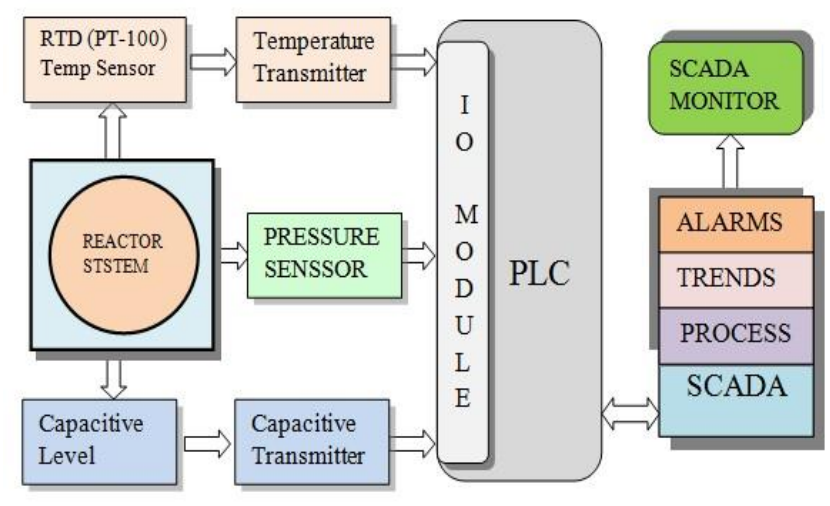

Fig. 1 Block Diagram of Proposed System

The hardware consist of the following are

1. Programmable Logic Controller

2. SCADA system with PC

3. Industrial chemical Reactor

4. Temperature sensor

5. Pressure sensor

6. Level Sensor

7. MODBUS Comm. Cable

A detailed explanation for each individual unit as present below

1. Programmable Logic Controller (PLC): The PLC used in the present work is Telemecanique Modicon Micro TSX PLC 3750 controller which has 16 digital inputs, 12 digital outputs 4 analog inputs and 2 analog outputs.

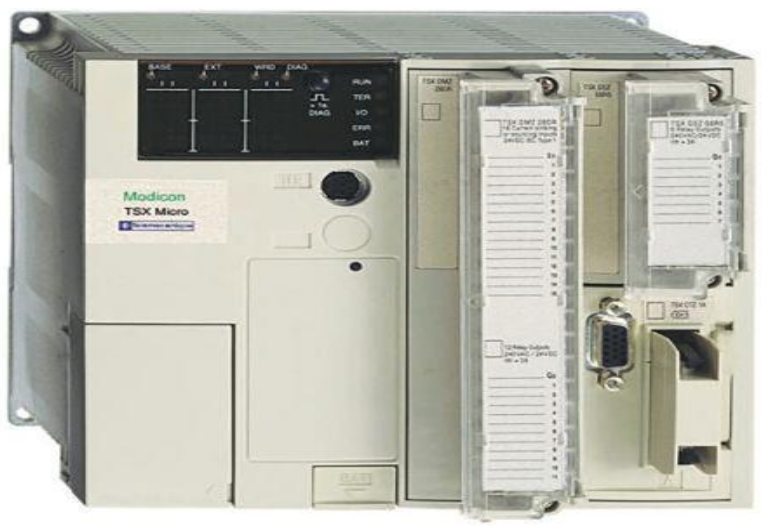

Fig.2 Programmable Logic Controller

The controller has various unique features such as (internal central processing unit (CPU) for processing typical electrical signals from the field sensors, The PLC has different type 412KB memories like Read Only Memory (ROM) for storing the firmware software and Random Access Memory(RAM) for user programmed memory. The PLC has multiple types of various interfacing protocols like MODBUS Serial communication protocol for communication with human machine interface (HMI), SCADA interface and various industrial devices. The PLC is programmed using ladder logic PL7 Pro V4.4 developing software
2. SCADA System with PC: The Supervisory Control And Data Acquisition (SCADA) is a purely software system, the present work SCADA software installed in personal computer with minimum configuration. The present personal computer has windows 7 operating system, 4GB RAM, 500GB hard disk and 2GB graphic card and etc. the SCADA system have unique features such as graphic design and development in multiple pages for the process visualization, developed the variable identification for individual memory location with different data types like integer, real, Word, and etc. in the SCADA developing own different type of libraries like genius, mages, and pages and etc. the system providing individual communication configuration with multiple devices. The SCADA system supported various communication protocols like MODBUS, PROFIBUS, Serial Comm., CANOPEN, MODNET and ETHERNET .The SCADA system communicate at a time multiple Programmable Logic Controllers (PLC) with various industrial protocols. The SCADA system basically alarms, trends, history and process popup, most automation industry used combination of PLC and SCADA both systems worked together information shared. In this system SCADA real-time measured and monitored of reactor process sensors continuously and stored the data in specific DATABASE of personal computer then present developed SCADA is VIJEO CITEC 2015 version 7.50 software

3. Industrial Chemical Reactor: For the preparation of bulk drug materials purpose and designed a prototype industrial chemical reactor, this reactor is made with stainless steel (SS-304) withstand temperature is above $300 \mathrm{deg}$ and internal mass maximum pressure of reactor up to 9 bar and isolated jacket pressure is 4 bar. The reactor system consisted of mass inlet and mass outlet as well as jacket inlet and jacket outlet as shown in fig.2. In the chemical reaction formation process having different type's temperature set point, so we need to meet the temperature set points then in the reactor jacket multiple types of temperature utilities derived. So prototype industrial chemical reactor without disturbing mass reaction continuously to measure and monitor the internal mass temperature of the reactor and also measure the derived utility temperature in the reactor jacket so for this purpose in the basic reactor design provided two provision of sensors placement. Any reactor system must have constant internal mass volume, this is decided that how much quantity of chemical drug having in the mass of the reactor then in the process of reaction. we must continuously measure and monitor the internal mass volume of the reactor, so for this purpose in design given to provision of level monitoring sensor, and also in reaction time process having internal mass reaching lower deg temperature to higher deg temperature the in the reactor developed a some constant pressure so for safety purpose continuously monitor the internal pressure of the prototype industrial chemical reactor 


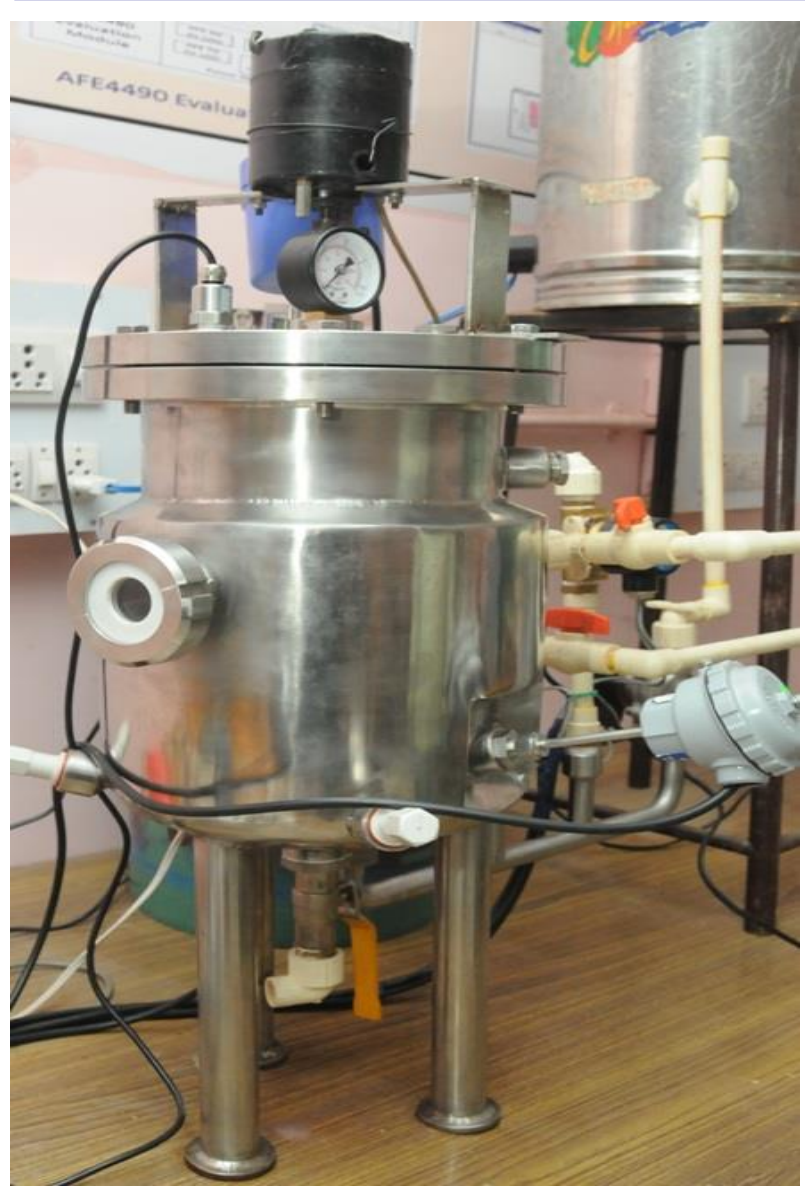

Fig.3 Chemical Reactor System

4. Resistive Temperature Detector (Pt-100): Resistive Temperature Detector (Pt-100) is used as a temperature sensor in the prototype industrial chemical reactor system the sensor range is $-50 \mathrm{deg}$ to $200 \mathrm{deg}$. In the RTD device having two types of measuring technique i.e. two wires and three wire sensors main difference is the accuracy increased in three wire sensors. The RTD sensor basic function is in the center of the reactor in reaction process internal mass temperature is continuously converted into change in resistance, the resistance is converted in to conventional 4-20 $\mathrm{mA}$ current signal with help of temperature transmitter.

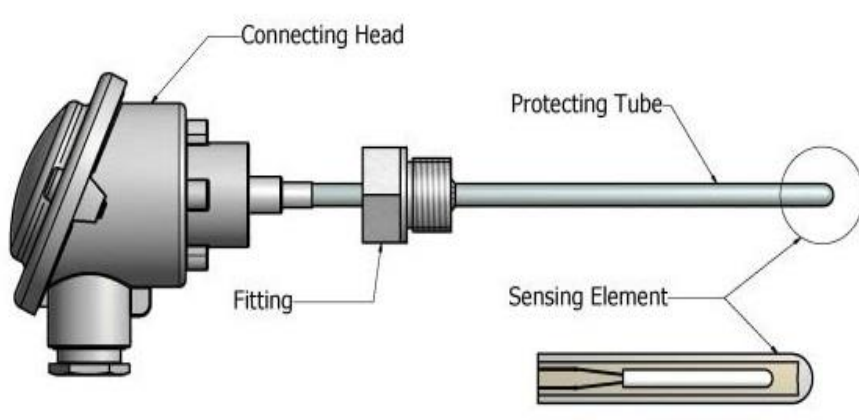

Fig. 4 Resistive Temperature Detector

The 4-20mA current connected with configured PLC analogto-digital input channel in I/O module of the PLC, the PLC process the current information to digital form then store the information in configured memory locations then processing the data in PLC processer
5. Capacitance Level Transmitter: Level measurement devices are many types in process industries like capacitance, ultrasonic, radar and hydrostatic level transmitters.

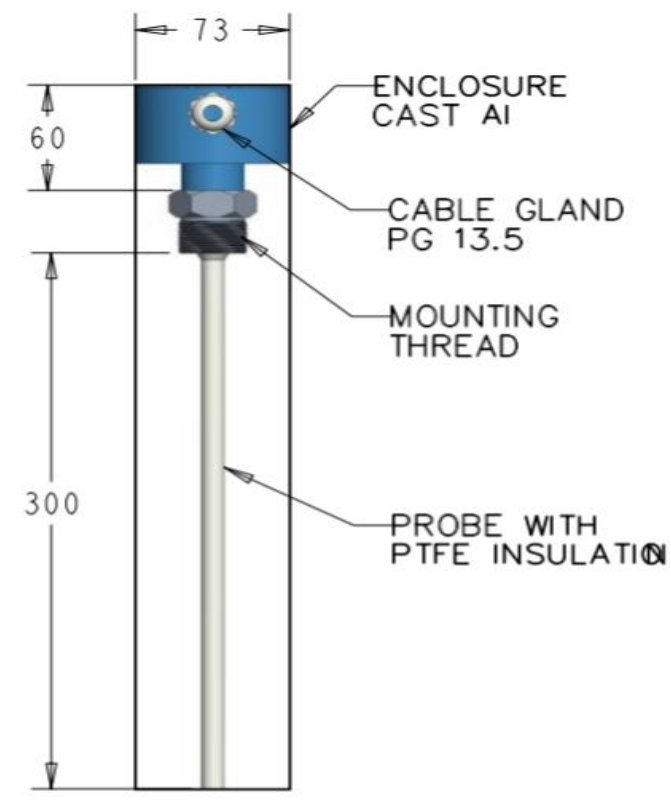

Fig.5 Capacitance Level Transmitter

In the prototype industrial chemical reactor system, the internal mass level measured with Capacitance Level Transmitter, the device is manufactured with stainless steel coated with Polytetrafluoroethylene (PTFE) used as probe material. The Capacitance Level Transmitter operating voltage is 12 to $30 \mathrm{vdc}$ power supply and it is with stands $-20 \mathrm{deg}$ to $120 \mathrm{deg}$ of operating temperature of reactor, the process of measuring level substances in reactor internal level ( 0 to $215 \mathrm{~mm}$ ) changing with simultaneously changed the capacitance of the device, The changed capacitance verses changing with output current $(4-20 \mathrm{~mA})$ of the transmitter. The conventional current connected to the configured analog-to-digital input channel of I/O module of the PLC. The PLC processing the digital information and then stored in to the dedicated memory location of PLC memory

\section{Diaphragm Pressure Transmitter:}

The diaphragm pressure sensor used to measure the prototype industrial chemical reactor internal mass pressure, the sensor made with stainless steel (SS316) and the sensor pressure range is $0-10$ bar with High Accuracy of $(+/-0.50 \%$ FSO $)$ then operating power supply is $24 \mathrm{~V} \mathrm{dc}$.

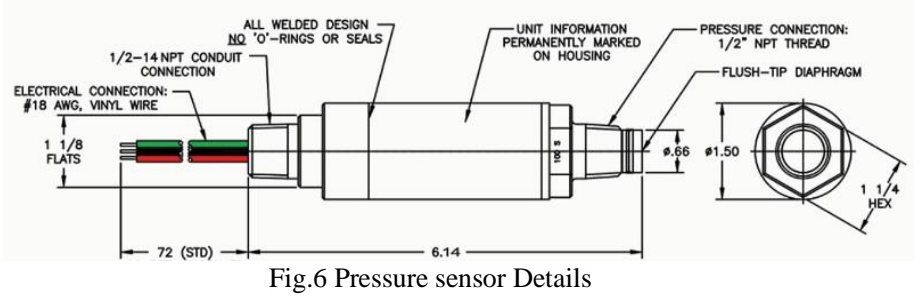

The main work function of this sensor is when the reaction in process in mass of the rector with differential pressure is developed so this pressure is continuously measured by the sensor, which provide the output in conventional electrical current 4-20mA. 


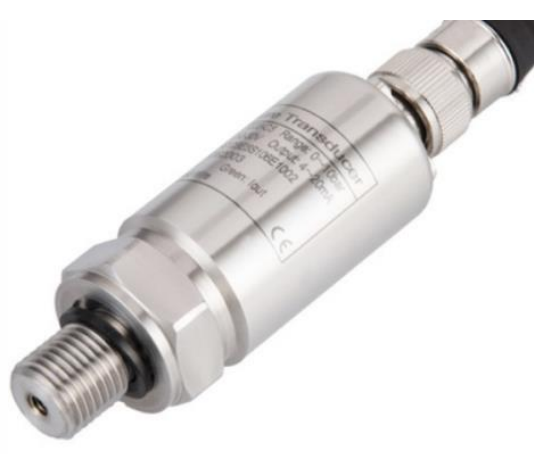

Fig. 7 Diaphragm Pressure Transmitter

The output of pressure sensor connected with the configured analog to digital channel of the PLC I/O module. The PLC module converted from electrical information to digital information and this data processed furthered by processer

7. MODBUS Serial Comm. Protocol Cable: The reactor system moving in process the sensor devices converting physical parameters in to electrical signal and are connected with the PLC then these signal convening into digital information data. The digital data processed by PLC processer in every scan cycle, the updated data need to visualized in SCADA system. In a modern technology device to device data communication is enabled in different types of protocols like Local area network (LAN), PROFIBUS, PROFINET, MODBUS, MODNET and etc.

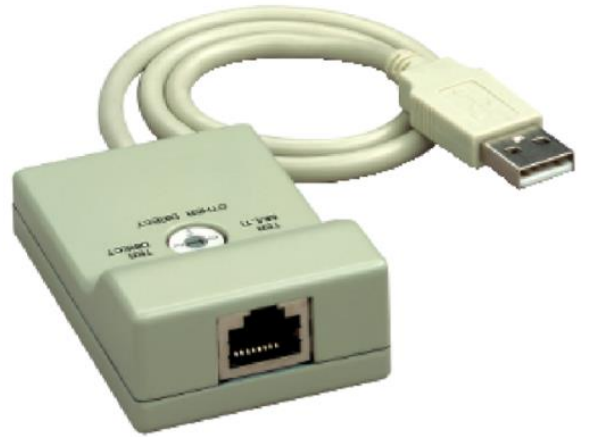

Fig. 8 Unitelway Comm. Cable

In the present system working on the MODBUS serial communication protocol from Personal computer SCADA system to PLC via TSX Micro's Telemecanique programming (Unitelway) cables TSXCUSB485, The TER port is physically the same port. The TER port is usually used for SCADA units via a protocol that supports multi dropping communications from plc to SCADA system.

\section{SOFTWARE IMPLIMENTATION}

The present Reactor process system consists of three physical parameters of mass temperature, level and internal pressure of rector system. For the measurement and monitoring of process system developed the PLC/SCADA system. The developing system used Telemecanique Modicon Micro TSX PLC 3750 controller for fetching the data from sensors of physical parameters of the system. The PLC programmed with PL7 Pro developing software. The configuration and development measurement techniques used as following parameters shown in below table.1

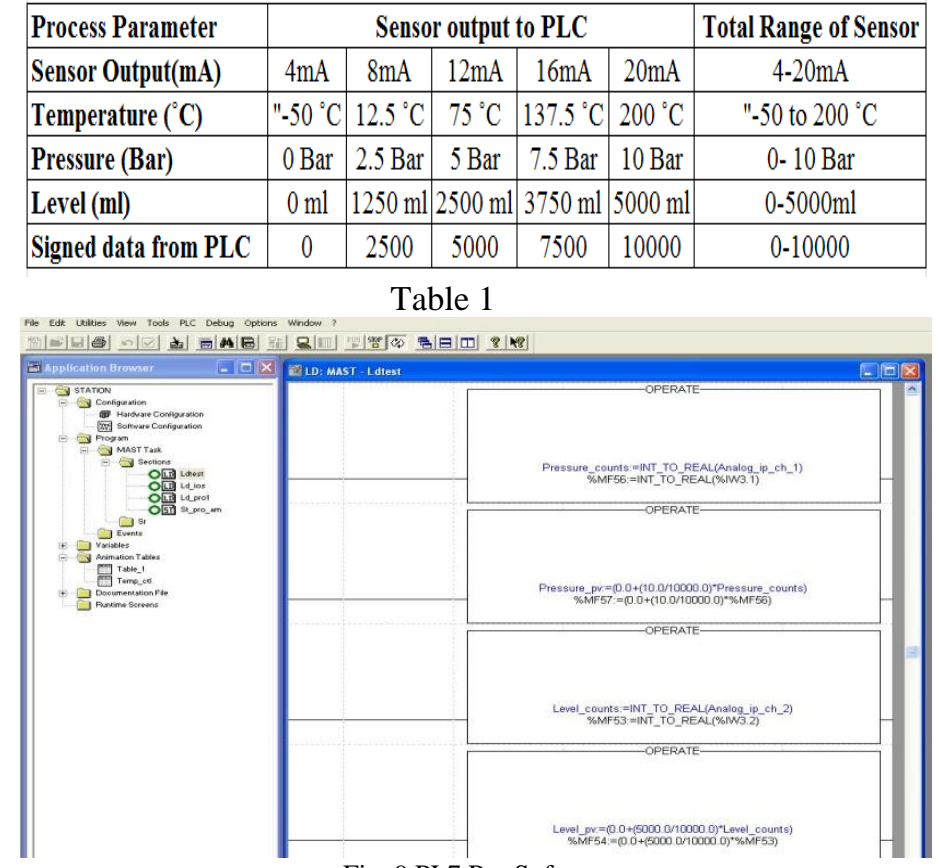

Fig. 9 PL7 Pro Software

The process parameters shown in graphical representation purpose used SCADA system of vijeo citect V7.5 designing software. This software contains different SCADA features that are process parameters numerically updated instant of time, process alert alarm system, parameters trending system, graphical visualized of measured parameters and etc. The reactor level and reactor volume measured and displayed in SCADA system in simulation way applied to the parameters also as shown in below fig. 10

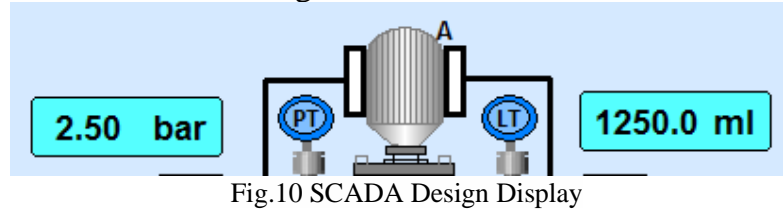

\section{RESULTS}

The system is successfully designed and developed for the reactor process parameters temperature, level and pressure is measured and monitored continuously. The complete reactor process sensors and actuators controlled by the programmable logic controller system, The PLC system converted analog data to required digital format. The digital information of PLC memory mapped with SCADA system memory location with MODBUS Serial communication protocol. The real-time numerical values of the Temperature, Level, and Pressure sensor information is graphical visualized in the SCADA system By selecting the Reactor button on the welcome screen then reactor screen will displayed shown in Fig. 11 


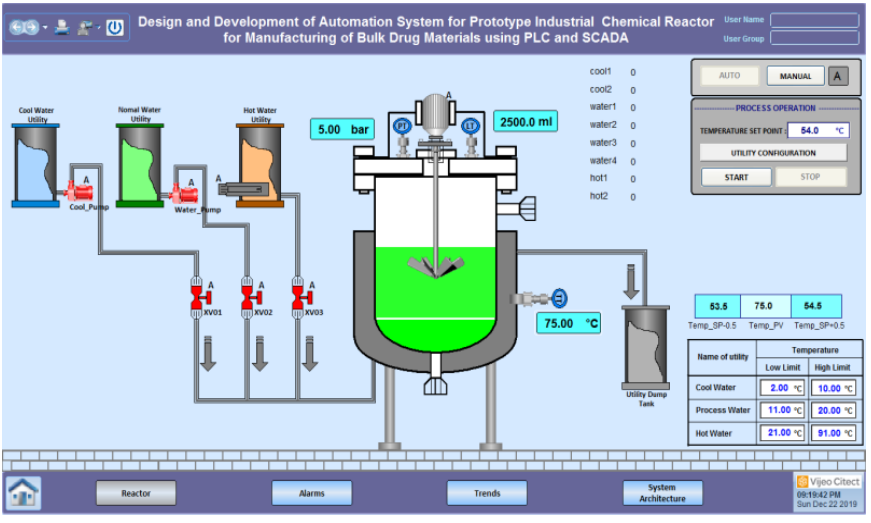

Fig.11 Numerical valuates Display

In the reactor screen number of graphical buttons designed, each button has specific function performed that they are opened popup windows. The window shown below process parameters like Temp_SP, Temp_PV and limits of the utility set points in numerical format shown in fig. 12

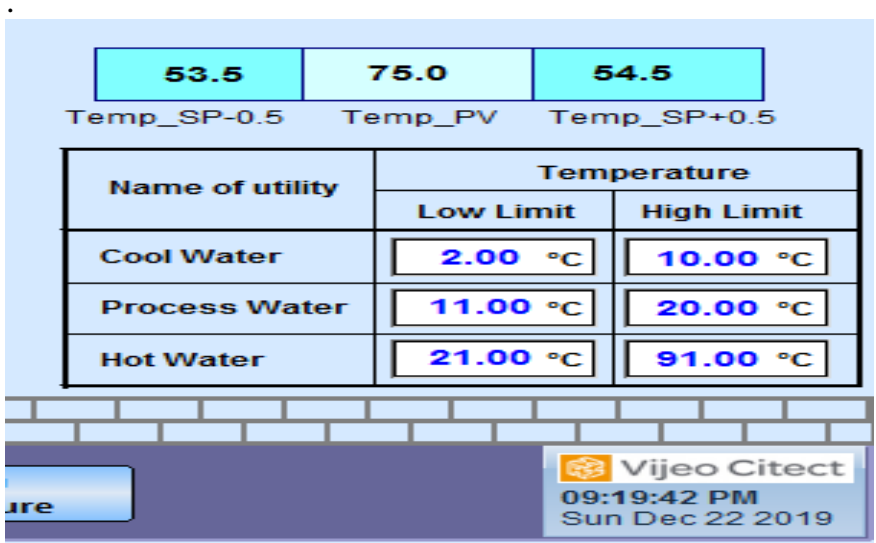

Fig.12 numerical values Display

In the SCADA system bottom of the screen selecting the trends button then displayed trends window shown the fig. 13 . This screen displayed process Parameters visual trends of temperature, level, pressure and set points of the controlled reactor process. In the each trend pen have different functions like Parameters units, instant storage of time interval, span range limits, color of the process plot and etc.

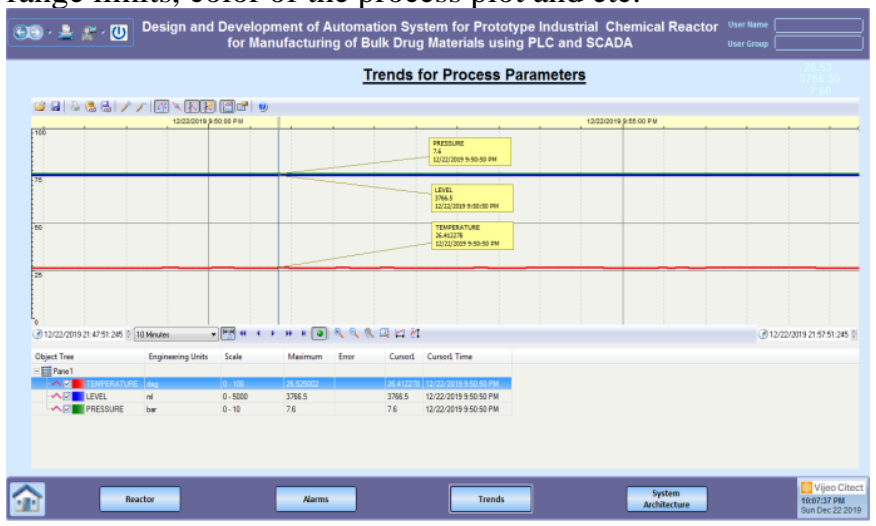

Fig.13 Parameters trends in SCADA

the SCADA system provided some of the advanced feature that's are reactor process parameters temperature ,level, pressure, set points, and etc continuously stored in the SCADA database with defined instant of time interval with respect of time. The graphical plot analysis of process parameter history in certain period of time, the SCADA system parameters values stored in the Database to Ex-cell table format with time and date of parameters shown in Fig. 14

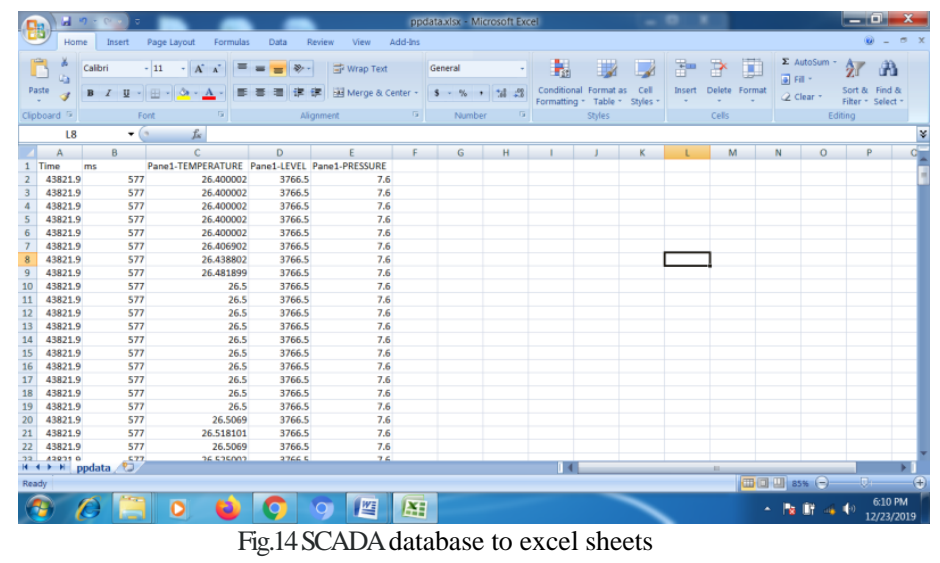

The complete reactor process alarms with time and date wise displayed in the alarm screen this is also sorting the system which alarm event present and past of the system shown in Fig. 15

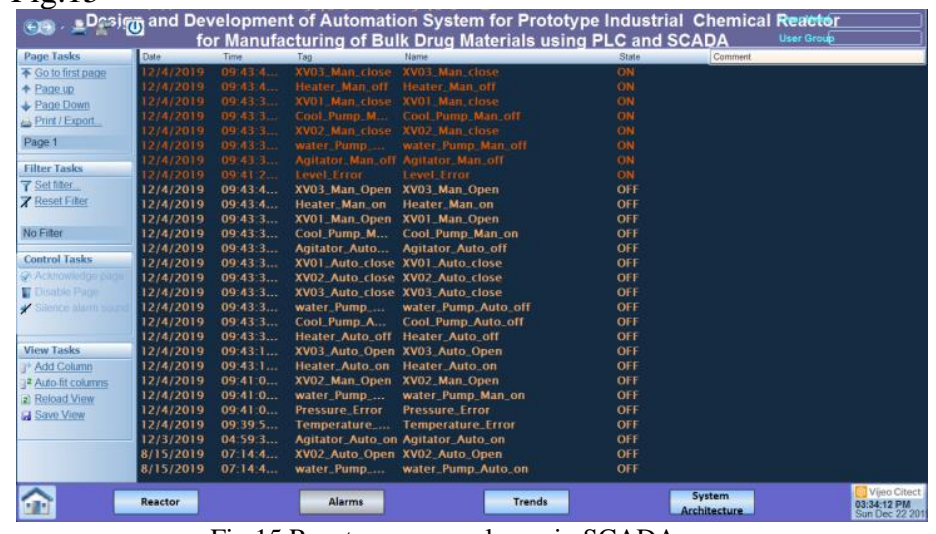

Fig.15 Reactor process alarms in SCADA

\section{CONCLUSION AND FUTURE SCOPE}

The present work developed on a real-time Programmable Logic Controller (PLC) and Supervisory Control And Data Acquisition (SCADA) based measurement and monitoring system. In the system PLC device has received information from sensor transmitter then information processed, analyzed and executed with predefined program instructions Then the Digital data stored in PLC memory. The PLC Device same as information shared with configured SCADA system via MODBUS comm. Protocol, in the SCADA system Received digital Data is linked with appropriate graphical images and numerical values displayed like temperature, level and pressure on Reactor system in SCADA. The system continuously stored sensors information in SCADA database, this is useful for the operator study, analysis and creating graphical trends history of the system. 


\section{REFERENCES}

[1] H. Kleines, J. Sarkadi, F. Suxdorf and K. Zwoll, "Measurement of real-time aspects of Simatic/spl reg/ PLC operation in the context of physics experiments," in IEEE Transactions on Nuclear Science, vol. 51, no. 3, pp. 489-494, June 2004. doi: 10.1109/TNS.2004.828504

[2] Li Wang and K. Liu, "Implementation of a Web-Based Real-Time Monitoring and Control System for a Hybrid Wind-PV-Battery Renewable Energy System," 2007 International Conference on Intelligent Systems Applications to Power Systems, Toki Messe, Niigata, 2007, pp. 1-6.

[3] Viswanathan, Rajeswari \& Suresh, L. \& Rajeshwari, Y.. (2013) Water storage and distribution system for pharmaceuticals using PLC and SCADA. Proceedings of IEEE International Conference on Circuit, Power and Computing Technologies, ICCPCT 2013. 79-86. 10.1109/ICCPCT.2013.6528951.

[4] S.Kalaivani, M.Jagadeeswari " PLC \& SCADA Based Effective Boiler Automation System for Thermal Power Plant" International Journal of Advanced Research in Computer Engineering \& Technology (IJARCET) Volume 4 Issue 4, April 2015

[5] K. Gowri Shankar " Control of Boiler Operation using PLC SCADA" Proceedings of the International Multi-Conference of Engineers and Computer Scientists 2008 Vol II IMECS 2008, 1921 March, 2008,Hong Kong.

[6] Sánchez Pacheco, Francisco \& Sotorrio, Pedro \& HerediaLarrubia, Juan \& Perez-Hidalgo, Francisco \& Sidrach-deCardona, M.. (2014). PLC-Based PV Plants Smart Monitoring System: Field Measurements and Uncertainty Estimation. Instrumentation and Measurement, IEEE Transactions on. 63. 2215-2222. 10.1109/TIM.2014.2308972.

[7] Promsawat, Thipichpon \& Kummool, Sart \& Pongswatd, Sawai \& Julsereewong, Amphawan. (2016). Real-time monitoring and reporting alarm system for $\mathrm{pH}$ measurement in wet scrubbers. 353-358. 10.1109/ICCAS.2016.7832343.

[8] Mumtaz, Fatima. (2015). Real-Time, PLC based, Energy Monitoring System. 1-5. 10.1109/INDICON.2015.7443747.

[9] Zaev, Emil \& Babunski, Darko \& Tuneski, Atanasko. (2016). SCADA system for real-time measuring and evaluation of river water quality. 83-86. 10.1109/MECO.2016.7525708.

[10] An Intelligent Model based level control of boiler Drum. KG Begum, D Mercy, HK Vedi, M Ramathilagam. International Journal of Emerging Technology and ...

[11] Irfan, Muhammad \& Saad, Nordin \& Ibrahim, Rosdiazli \& Asirvadam, Vijanth. (2013). Development of an intelligent condition monitoring system for AC induction motors using PLC. BEIAC 2013 - 2013 IEEE Business Engineering and Industrial Applications Colloquium. 789-794. 10.1109/BEIAC.2013.6560243.

[12] Ramasami, Ashok \& Pandimeena, S \& Rajameena, R \& Jeyanthi, R \& Ramya, S. (2016). Modern Industrial Power Plant Boiler Automation Using GSM Technology.

[13] Fuzzy Logic Approach for Boiler Temperature \& Water Level Control A Shome, SD Ashok - International Journal of Scientific \& Engineering ..., 2012

[14] Moursi, Israa \& El-Din, Loay. (2014). SCADA system for oil refinery control. Measurement. 47. 5-13. 10.1016/j.measurement.2013.08.032.

[15] Lakhoua, Mohamed Najeh. (2010). SCADA applications in thermal power plants. International Journal of Physical Sciences. 5. $1175-1182$.

[16] Xibin, Wang \& Guohong, Li \& Xuejie, Wei. (2011). PLC-based SCADA system for oil storage and application. 10.1109/ICEICE.2011.5777205

[17] Wiliem, Leonard \& Hargreaves, Douglas \& Stapelberg, Rudolph \& Yarlagadda, Prasad. (2007). Development of Real-Time Data Filtering for SCADA System. Journal of Achievements in Materials and Manufacturing Engineering. 21

[18] U. Younas, S. Durrani and Y. Mehmood, "Designing Human Machine Interface for Vehicle's EFI Engine Using Siemen's PLC and SCADA System," 2015 13th International Conference on Frontiers of Information Technology (FIT), Islamabad, 2015, pp. 205-210. doi: 10.1109/FIT.2015.44
[19] Yang, X.H., 2014. Design and Research for a Boiler Steam Drum Control System Based on PLC. Advanced Materials Research $1030-1032,1442-1444$ https://doi.org/10.4028/www.scientific.net/amr.1030-1032.1442

[20] Das, Rishabh, Sayantan Dutta, Anusree Sarkar and Kaushik Samanta. "Automation of Tank Level Using Plc and Establishment of Hmi by Scada." (2013).

[21] R. P. Jerrard, "Temperature Drop to Resistance Temperature Detector in Stator Windings of Turbine Generators [includes discussion]," in Transactions of the American Institute of Electrical Engineers. Part III: Power Apparatus and Systems, vol. 73, no. $1, \quad$ pp. 665-670, Jan. 1954.doi:10.1109/AIEEPAS.1954.4498872

[22] M. Laciak, P. Fazekaš and J. Kačur, "Monitoring and control of temperatures in electric furnace in PROMOTIC system," 2011 12th International Carpathian Control Conference (ICCC), Velke Karlovice, 2011, pp. 239-242. doi:10.1109/CarpathianCC.2011.5945855

[23] S. Pradhan and S. Sen, "An improved lead compensation technique for three-wire resistance temperature detectors," in IEEE Transactions on Instrumentation and Measurement, vol 48, no. 5, pp. 903-905, Oct. 1999. doi: 10.1109/19.799644

[24] T. K. Maiti, "A Novel Lead-Wire-Resistance Compensation Technique Using Two-Wire Resistance Temperature Detector," in IEEE Sensors Journal, vol. 6, no. 6, pp. 1454-1458, Dec. 2006. doi: 10.1109/JSEN.2006.883903 